Ann. Biol. anim. Bioch. Biophys., I970, 10 (2), 223-238.

\title{
HÉTÉROGONIE BIOCHIMIQUE DES COMPOSANTS AZOTÉS DU GRAND PSOAS AU COURS DE LA GROISSANCE POST-NATALE DU LAPIN
}

\author{
R. BARON et P. VIGNERON \\ Station de Physiologie animale, \\ Centre de Recherches de Montpellier \\ École nationale supérieure agronomique de Montpellier \\ Institut national de la Recherche agronomique
}

\section{RÉSUMÉ}

La composition du grand psoas a été étudiée chez des lapins mâles et femelles d’âge échelonné entre 3 et 26 semaines.

Cette étude a porté sur les teneurs en azote des fractions suivantes :

- Azote myofibrillaire

- Azote sarcoplasmique

-. Azote du stroma

- Azote non protéique

- déterminées par la technique de Helander - ainsi que sur lạ teneur en matière sèche du muscle.

Il ressort que :

-. la teneur en matière sèche augmente au cours de la vie, mais évolue différemment dans les deux sexes; le Lapin

— les teneurs relatives en azote des différentes fractions semblent fixées dès 3 semaines chez

- il existe une différence faible mais significative dans les teneurs en azote du grand psoas d'animaux mâles et femelles, les mâles présentant une teneur plus importante en azote myofibrillaire et plus faible en azote sarcoplasmique.

\section{INTRODUCTION}

Les modalités de la croissance pondérale globale et relative du Lapin domestique sont maintenant mieux connues (CANTIER et al., I969). Nous avons donc jugé nécessaire d'entreprendre l'étude des phénomènes biologiques et biochimiques qui participent au développement des tissus et organes dans cette espèce. 
La constitution biochimique du muscle est depuis longtemps l'objet de nombreux travaux mais n'a pas encore été totalement élucidée. De plus, les chercheurs se sont surtout intéressés à la composition du muscle, à un âge donné, dans le cadre d'études sur la physiologie de la contraction musculaire ou sur l'évolution biochimique postmortem de la viande. Certains auteurs, plus rares, se sont consacrés à l'étude de l'évolution quantitative des diverses catégories de protéines musculaires au cours de la croissance. On peut citer : RobInson (I952), Mrzuno et al. (I967) chez le Poussin; Dickerson (I960), Moss et al. (I964, I967) chez le Poulet; ScharpF et al. (I964) chez le Dindon; Gordon et al. (r967), Herrmann et Nicholas (I948), NeEdHam (I93 I) chez le Rat; CREPAX (I952), IVANov (I967) chez le Lapin ; Lawrie (I963) chez les bovins ; Dickerson et Widnowson (I960) chez le Porc et l'Homme.

Cependant, n'ont été étudiées ni les différences entre sexes, ni l'influence de l'évolution de la teneur en eau du tissu musculaire sur les concentrations respectives de ces diverses catégories de protéines. C'est dans cette perspective que ce travail a été envisagé chez le Lapin.

\section{I. - MATÉRIEL E'T MÉTHODES}

\section{Matériel animal}

Les échantillons ont été prélevés sur une souche de lapins communs âgés de : 3 semaines, 5 semaines 2 jours, 7 semaines 2 jours, 12 semaines 2 jours, 16 semaines, 20 semaines et 26 semaines. Les conditions d'élevage ont été décrites par Prud'hon (i 967 ).

Les animaux ont été sacrifiés après avoir été assommés.

Nous avons étudié l'évolution de la concentration en azote de diverses fractions dans un muscle unique : le Grand Psoas, choisi pour les raisons suivantes :

- de taille importante il est facile à prélever rapidement et entièrcment, l'opération pouvant s'effectuer en moins de 5 minutes y compris le temps de saignée;

- il est dépourvu d'intersections aponévrotiques; on peut donc espérer une bonne homogénéité de la composition de ses diverses portions, à un niveau donné sur une section transversale;

- enfin, c'est un muscle dont l'allométrie de croissance cst très voisine de celle de la masse musculaire totale (CANTIER et al,, en préparation).

\section{Méthodes chimiques}

Le protocole expérimental est celui mis au point par HELANDER (I957).

Un muscle entier ayant été refroidi dans un congélateur, en ro minutes à - $15^{\circ} \mathrm{C}$, des coupes en série sont pratiquées, à l'aide d'un microtome, dans une chambre froide à $2^{\circ} \mathrm{C}$. Les échantillons ont été prélevés dans la partie médiane du muscle, la teneur en lipides pouvant varier à l'intérieur d'un même muscle. Les protéines sont extraites d'échantillons d'un poids de 0,2 à $0,3 \mathrm{~g}$.

Cette technique, fondée sur l'extraction différentielle des protéines musculaires à l'aide de solutions tamponnées de forces ioniques différentes, permet de discriminer quatre catégories d'azote dans l'azote total musculaire (tabl. I) : bine) ;

- azote non protéique ;

- azote des protéines sarcoplasmiques (myogènes, myoalbumine, globulines X, myoglo-

- azote des protéines myofibrillaires (protéines de structure ou protéines contractiles : myosine, actine, actomyosine, tropomyosine);

- azote du stroma.

Les dosages ont été faits par semi-micro Kjehldahl. 


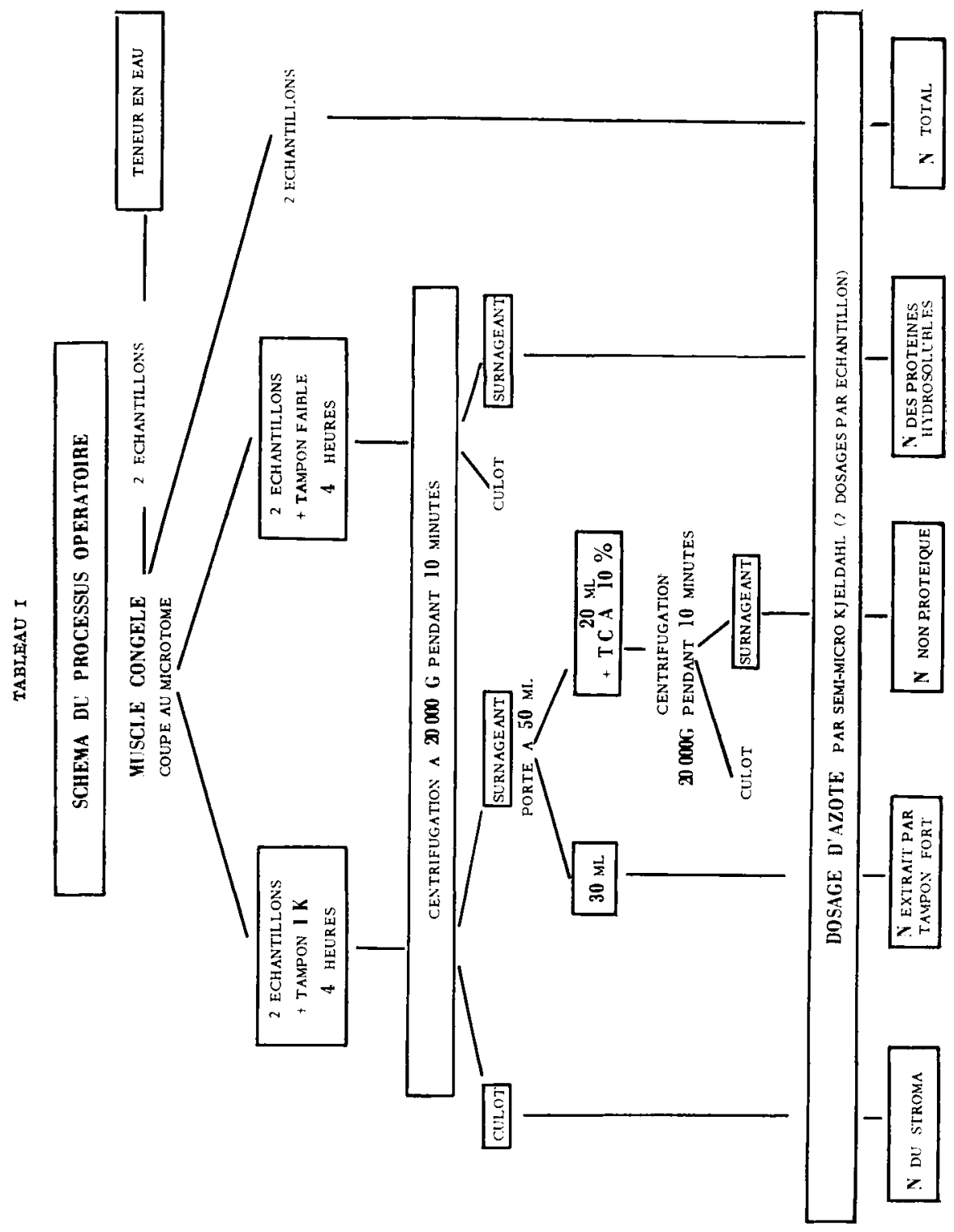




\section{Méthodes mathématiques}

a) Choix des animaux.

La répétabilité des mesures a été estimée par leur coefficient de fidélité (Rouvier, I965) qui est le coefficient de corrélation intraclasse entre deux répétitions d'une mesure. Compte tenu du degré de précision souhaité, 5 animaux par stade et par sexe ont permis d'observer une variabilité inférieure à $8 \mathrm{p}$. Ioo pour chaque constituant étudié (DAGNELIE, I964). Le tableau 2 explicite les catégories étudiées.

\section{TABLEAU 2}

\section{Définition des catégories étudiées}

$\begin{array}{ll}\text { Poids vif en grammes } & \mathrm{X}_{1} \\ \text { Teneur en matière sèche (p. Ioo) } \mathrm{X}_{9} \\ \text { Temps en jours } & \mathrm{X}_{10}\end{array}$

\begin{tabular}{|c|c|c|}
\hline Constituants protéiques & $\begin{array}{l}\text { mg d'azote } \\
\text { par g } \\
\text { de tissu frais } \\
\text { (mesuré) }\end{array}$ & $\begin{array}{c}\text { mg d'azote } \\
\text { par g } \\
\text { de tissu sec } \\
\text { (calculé) }\end{array}$ \\
\hline $\begin{array}{l}\text { Azote total }{ }_{1} \ldots \ldots \ldots \\
\text { Azote extrait par I } \mathrm{K} \ldots \ldots \ldots \ldots \ldots \ldots \\
\text { Azote extrait par tampon faible } \\
\text { Azote non protéique } \ldots \ldots \ldots \ldots \ldots \ldots \ldots \\
\text { Azote du stroma } \ldots \ldots \ldots \ldots \ldots \ldots \ldots \\
\text { Azote des protéines myofibrillaires } \ldots \ldots \ldots \\
\text { Azote des protéines sarcoplasmiques }\end{array}$ & $\begin{array}{l}X_{2} \\
X_{3} \\
X_{4} \\
X_{5} \\
X_{6} \\
X_{7} \\
X_{8}\end{array}$ & $\begin{array}{l}X_{11} \\
X_{12} \\
X_{13} \\
X_{14} \\
X_{15} \\
X_{16} \\
X_{17}\end{array}$ \\
\hline 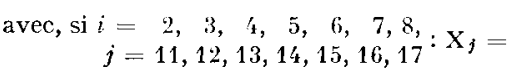 & $\mathrm{K}_{\ell} \times \frac{100}{\mathrm{X}_{\mathrm{g}}}$ & \\
\hline
\end{tabular}

b) Analyse de variance en fonction d'un facteur contrôlé (temps).

Le programme unfak, dû à Millier (I968) a donné les résultats des tableaux $3 \mathrm{~A}$ et $3 \mathrm{~B}$. Dans le cadre de cette étude, ce programme a l'avantage de condenser l'information contenue dans chaque stade, pour chaque catégorie, et pour chaque sexe, en une moyenne et un écarttype.

c) Méthode d'étude de l'évolution des caractères biochimiques.

Brody (1945) remarque que les " organismes changent sur le plan géométrique afin de demeurer les mêmes sur le plan physiologique ". A partir de ce principe Gunther et GuERRA (1955, I957), STAhL (I957), relient les variables physiologiques au poids du corps par la fonction puissance, comme l'avait fait NeEdham (I954) pour les variables biochimiques.

C'est ainsi que chaque catégorie $\mathrm{X} i$ a été ajustée au poids vif selon l'équation de régression :

$$
\log \mathrm{X}_{i}=a+b \log \left(\mathrm{X}_{1}\right)+c\left(\log \mathrm{X}_{1}\right)^{2}
$$

Cette formule parabolique tient compte des nombreuses tendances curvilignes observées dans les représentations hétérogoniques. Ce mode de représentation permet de suivre l'évolution des variations relatives des concentrations de chaque fraction azotée au cours de la croissance. 


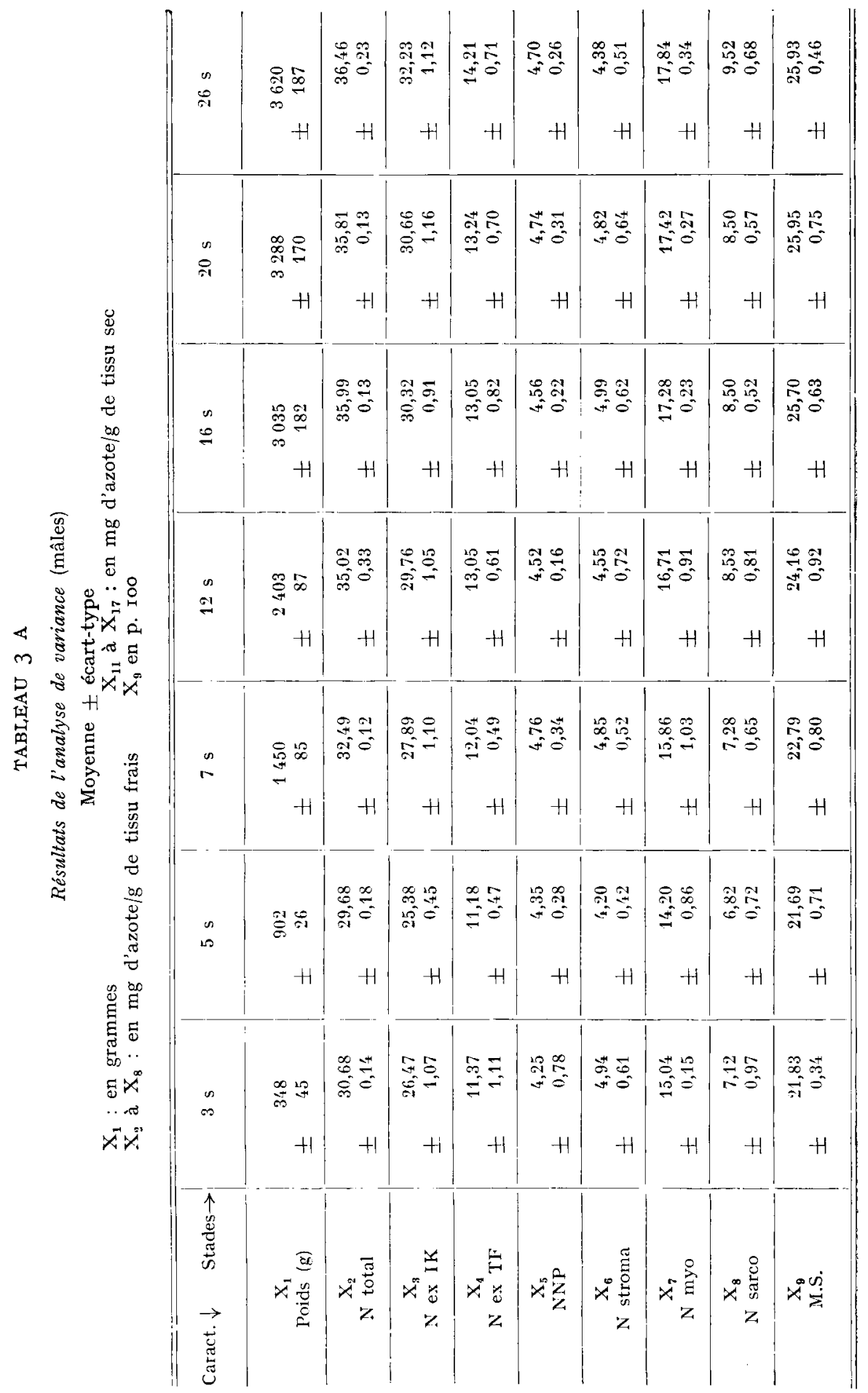


R. BARON, P. VIGNERON

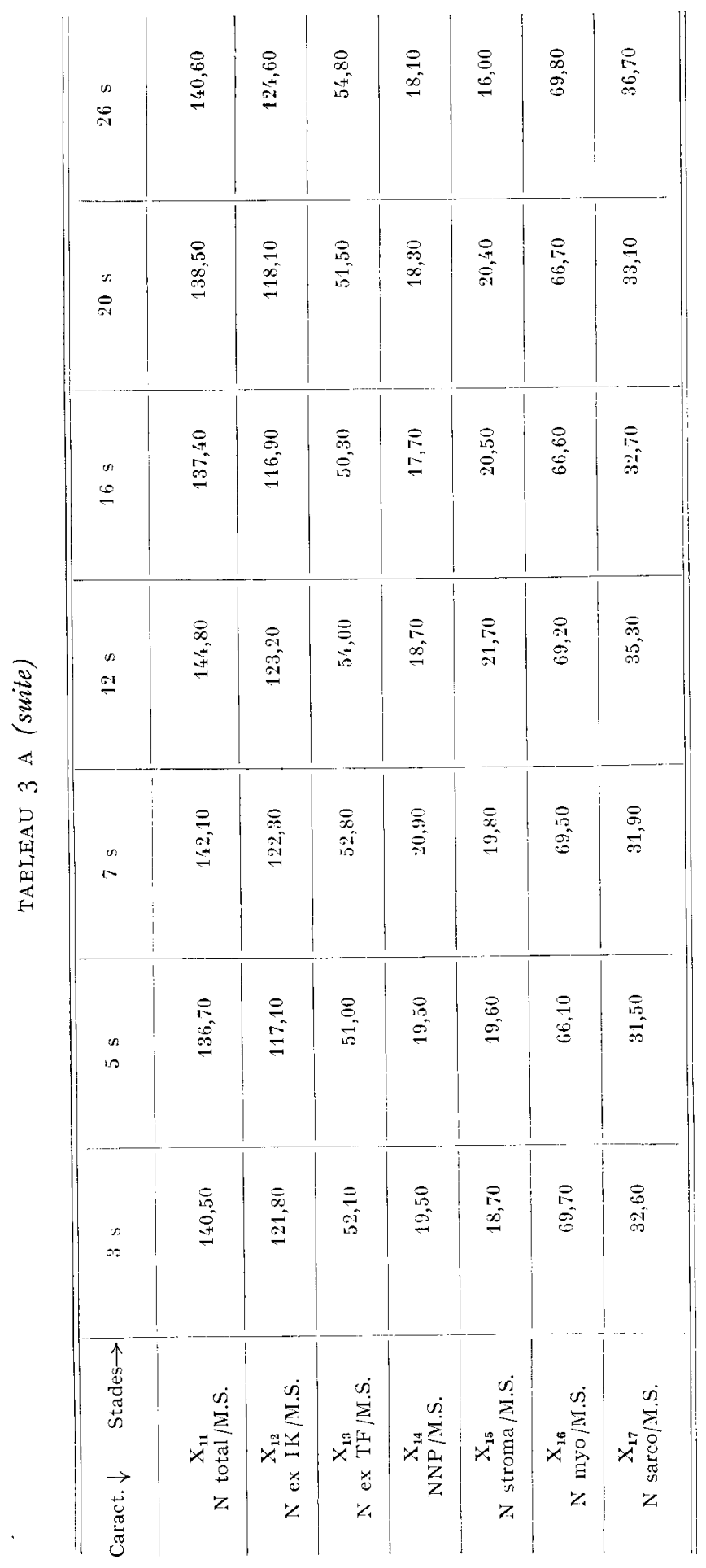




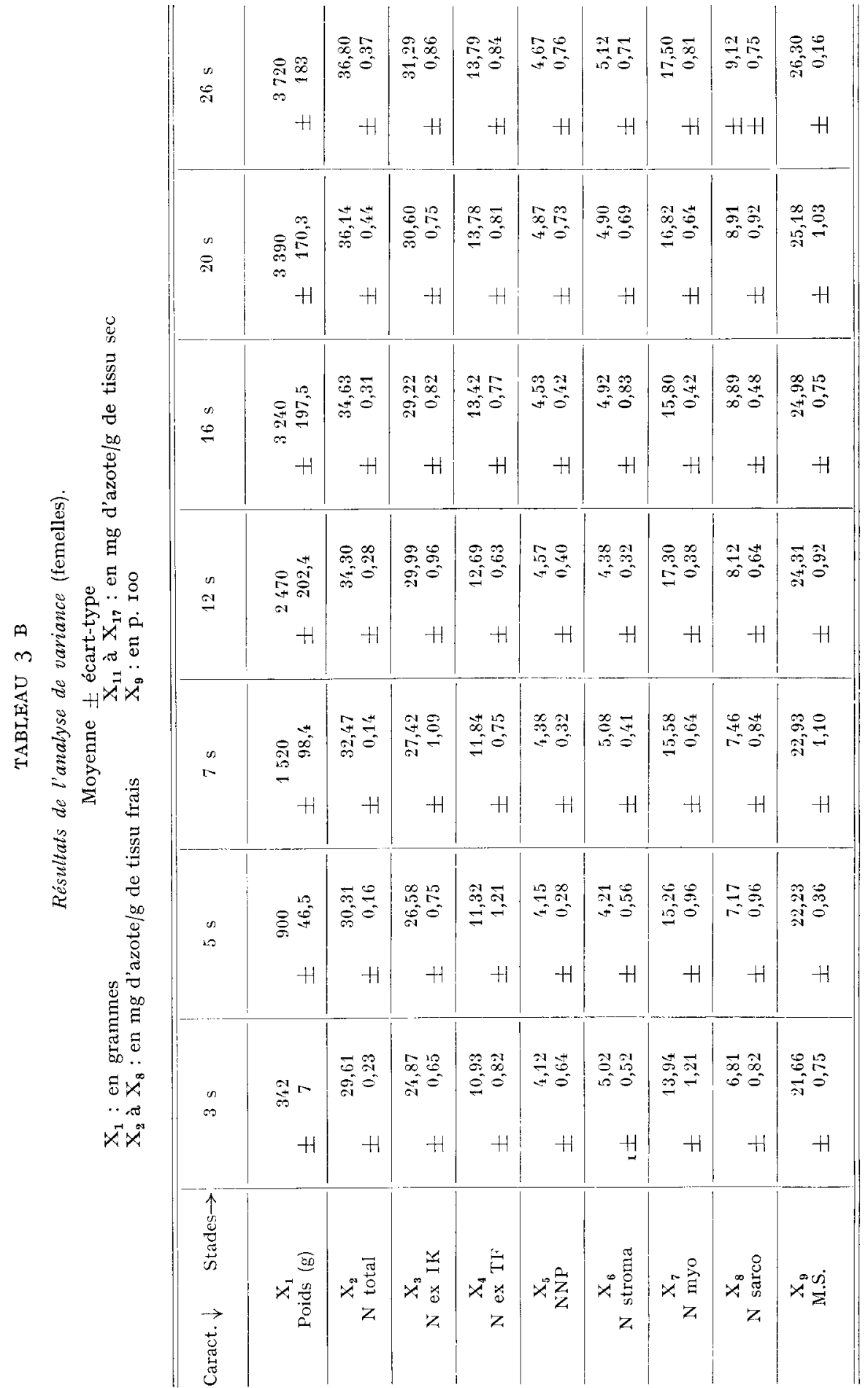




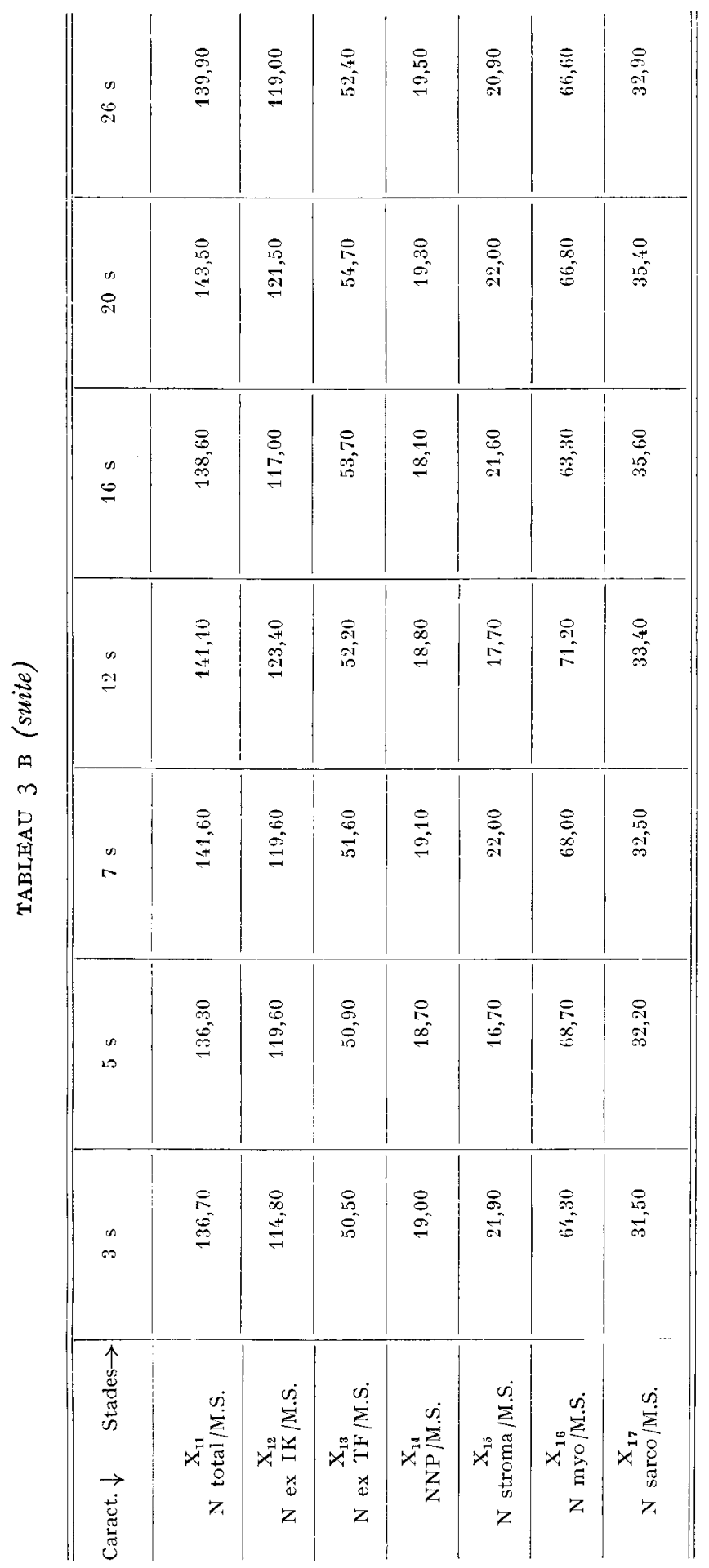


En effet, si la pente de la tangente à la courbe est supérieure à 1 le rapport $\frac{\mathrm{X}_{i}}{\mathrm{X}_{1}}$ augmente avec $\mathrm{X}_{1}$ :

$$
\begin{aligned}
\frac{\mathrm{d}\left(\log \mathrm{X}_{i}\right)}{\mathrm{d}\left(\log \mathrm{X}_{1}\right)}=\frac{\frac{\mathrm{dX}_{i}}{\mathrm{X}_{i}}}{\frac{\mathrm{dX_{1 }}}{\mathrm{X}_{1}}}>1 \rightarrow \frac{\mathrm{dX}_{i}}{\mathrm{X}_{i}}>\frac{\mathrm{dX_{1 }}}{\mathrm{X}_{1}} \\
\frac{\mathrm{dX}_{i}}{\mathrm{~d} \mathrm{X}_{1}}>\frac{\mathrm{X}_{i}}{\mathrm{X}_{1}}
\end{aligned}
$$

ou :

\section{TABLEAU 4 A}

Equation de régression $\log \mathrm{X}_{i}=\mathrm{a}+\mathrm{b} \log \mathrm{X}_{1}+\mathrm{c}\left(\log \mathrm{X}_{1}\right)^{2}$. Mâles $i=2,3,4,5,6,7,8, \mathrm{II}, \ldots \mathrm{I} 7 \quad r_{1}=$ coeff. corrél. totale $\left(\log \mathrm{X}_{i}, \log \mathrm{X}_{1}\right)$ $\mathrm{R}^{2}=$ coefficient de corrélation multiple $r_{2}=$ coeff. corrél. totale $\left(\log \mathrm{X}_{i},\left(\log \mathrm{X}_{1}\right)^{2}\right.$

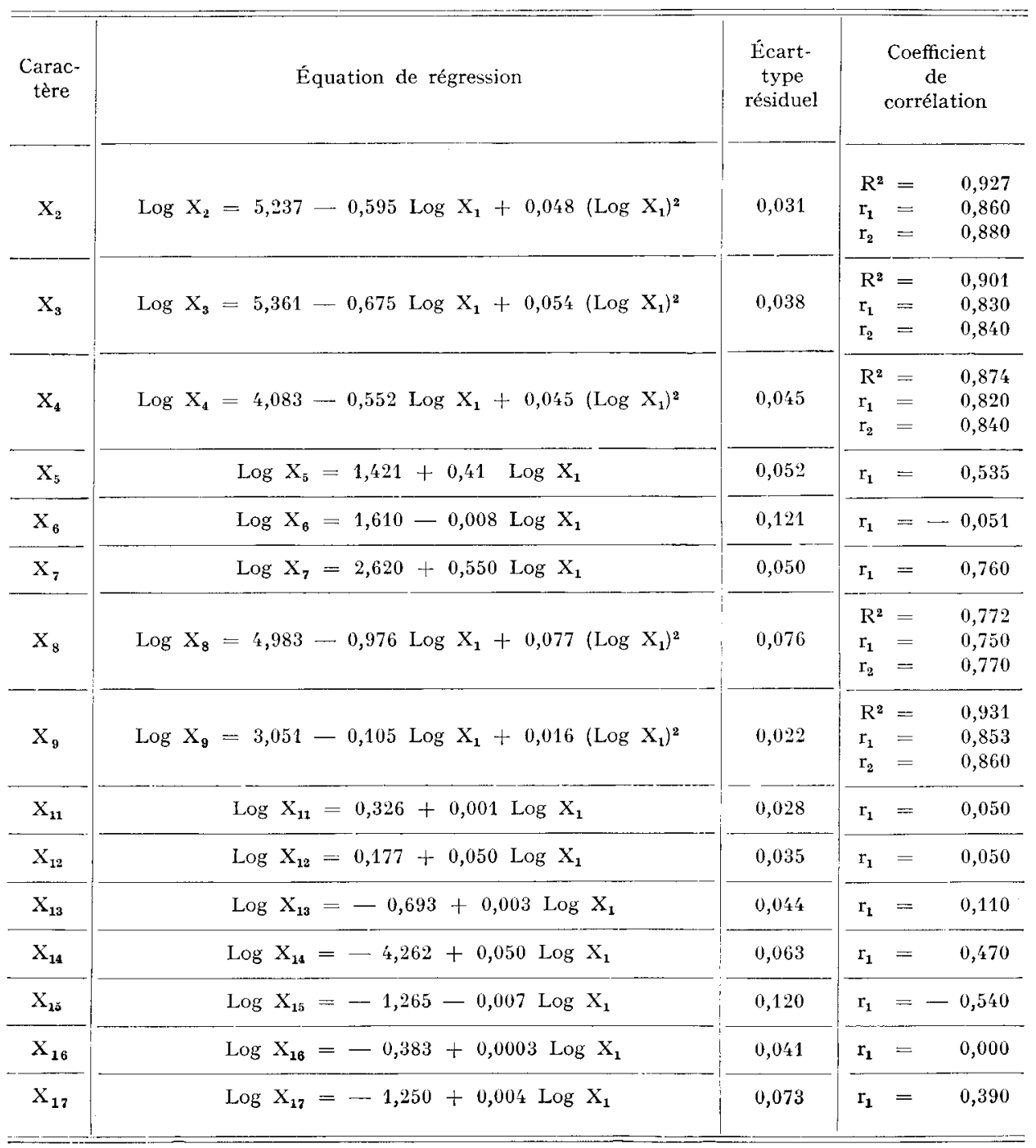


La justification théorique de ce modèle polynomial sera précisée dans un autre article (BARON, Rouvier, en préparation).

L'ajustement a été réalisé grâce à un programme de régression progressive multiple (REPRG dû à Tomassonne, 1967) dont les résultats sont exposés aux tableaux $4 \mathrm{~A}$ et $4 \mathrm{~B}$.

\section{TABLEAU 4 B}

Équation de régression $\log \mathrm{X}_{i}=\mathrm{a}+\mathrm{b} \log \mathrm{X}_{1}+\mathrm{c}\left(\log \mathrm{X}_{1}\right)^{2}$. Femelles $i=2,3,4,5,6,7,8, \mathrm{II}, \ldots \mathrm{I} 7 \quad r_{1}=$ coeff. corrél. totale $\left(\log \mathrm{X}_{i}, \log \mathrm{X}_{1}\right)$ $\mathrm{R}^{2}=$ coefficient de corrélation multiple $r_{2}=$ coeff. corrél. totale $\left(\log \mathrm{X}_{i},\left(\log \mathrm{X}_{1}\right)^{2}\right)$

\begin{tabular}{|c|c|c|c|c|c|}
\hline $\begin{array}{l}\text { Carac- } \\
\text { tère }\end{array}$ & Équation de régression & $\begin{array}{l}\text { Écart- } \\
\text { type } \\
\text { résiduel }\end{array}$ & \multicolumn{3}{|c|}{$\begin{array}{l}\text { Coefficient } \\
\text { de } \\
\text { corrélations }\end{array}$} \\
\hline $\mathrm{X}_{2}$ & $\log X_{2}=4,400-0,360 \log X_{1}+0,032\left(\log X_{1}\right)^{2}$ & 0,020 & $\begin{array}{l}\mathrm{R}^{2} \\
\mathrm{r}_{1} \\
\mathrm{r}_{2}\end{array}$ & $\begin{array}{l}= \\
= \\
=\end{array}$ & $\begin{array}{l}0,969 \\
0,940 \\
0,950\end{array}$ \\
\hline $\mathrm{X}_{\mathbf{3}}$ & $\log X_{3}=3,486-0,147 \log X_{1}+0,017\left(\log X_{1}\right)^{2}$ & 0,027 & $\begin{array}{l}\mathrm{R}^{2} \\
\mathrm{r}_{1} \\
\mathrm{r}_{2}\end{array}$ & $\begin{array}{l}= \\
= \\
=\end{array}$ & $\begin{array}{l}0,948 \\
0,940 \\
0,940\end{array}$ \\
\hline $\mathrm{X}_{4}$ & $\log X_{4}=4.019-0,561 \log X_{1}+0,047\left(\log X_{1}\right)^{2}$ & 0,040 & $\begin{array}{l}\mathrm{R}^{2} \\
\mathrm{r}_{1} \\
\mathrm{r}_{2}\end{array}$ & $\begin{array}{l}= \\
= \\
=\end{array}$ & $\begin{array}{l}0,921 \\
0,880 \\
0,890\end{array}$ \\
\hline $\mathrm{X}_{5}$ & $\log \mathrm{X}_{5}=1,235+0,0046\left(\log \mathrm{X}_{1}\right)^{2}$ & 0,074 & $\begin{array}{l}\mathrm{R}^{2} \\
\mathrm{r}_{2}\end{array}$ & $\begin{array}{l}= \\
=\end{array}$ & $\begin{array}{l}0,606 \\
0,590\end{array}$ \\
\hline $\mathrm{X}_{6}$ & $\log X_{6}=1,420-0,230 \log X_{1}$ & 0,098 & $r_{1}$ & $=$ & 0,050 \\
\hline$x_{7}$ & $\log x_{7} \Rightarrow 2,650+0,547 \log x_{1}$ & 0,052 & $r_{1}$ & $=$ & 0,790 \\
\hline $\mathrm{X}_{8}$ & $\log X_{8}=3,867-0,658 \log X_{1}+0,055\left(\log X_{1}\right)^{2}$ & 0,073 & $\begin{array}{l}\mathrm{R}^{2} \\
\mathrm{r}_{1} \\
\mathrm{r}_{2}\end{array}$ & $\begin{array}{l}= \\
= \\
=\end{array}$ & $\begin{array}{l}0,833 \\
0,790 \\
0,800\end{array}$ \\
\hline $\mathrm{X}_{9}$ & $\log \mathrm{X}_{9}=2,322+0,102 \log \mathrm{X}_{1}+0,0015\left(\log \mathrm{X}_{1}\right)^{2}$ & 0,031 & $\begin{array}{l}R^{2} \\
r_{1} \\
r_{2}\end{array}$ & $\begin{array}{l}= \\
= \\
=\end{array}$ & $\begin{array}{l}0,942 \\
0,770 \\
0,790\end{array}$ \\
\hline $\mathrm{X}_{11}$ & $\log X_{11}=0,232+0,013 \log X_{1}$ & 0,024 & $\mathrm{r}_{1}$ & $=$ & 0,41 \\
\hline $\mathrm{X}_{12}$ & $\log x_{12}=0,041+0,018 \log X_{1}$ & 0,035 & $r_{1}$ & $==$ & 0,37 \\
\hline$X_{13}$ & $\log X_{13}=-0,776+0,002 \quad \log X_{1}$ & 0,045 & $r_{1}$ & $=$ & 0,50 \\
\hline $\mathrm{X}_{14}$ & $\log X_{14}=-1,630+0,0008 \log X_{1}$ & 0,080 & $\mathrm{r}_{1}$ & $=$ & 0,11 \\
\hline $\mathrm{X}_{15}$ & $\log X_{15}=-1,117-0,068 \log X_{1}$ & 0,098 & $\mathrm{r}_{1}$ & $=$ & 0,50 \\
\hline $\mathrm{X}_{16}$ & $\log X_{16}=-0,454+0,006 \quad \log X_{1}$ & 0,061 & $\mathrm{r}_{\mathbf{1}}$ & $=$ & 0,09 \\
\hline $\mathrm{X}_{17}$ & $\log X_{17}=-1,294+0,003 \quad \log X_{1}$ & 0,074 & $\mathbf{r}_{\mathbf{I}}$ & $=$ & 0,47 \\
\hline
\end{tabular}




\section{II. - RÉSULTATS}

Les résultats sont rassemblés dans les tableaux $3 \mathrm{~A}, 3 \mathrm{~B}$ et exprimés en $\mathrm{mg}$ d'azote par g de tissu frais ou de tissu sec. L'évolution des concentrations des différentes formes azotées en fonction du poids vif en $\mathrm{g}$ est figurée en coordonnées arithmétiques (fig. I A, I B) et logarithmiques (fig. $2 \mathrm{~A}, 2 \mathrm{~B}$ ). On a également représenté l'évolution de la teneur en matière sèche du psoas au cours de la croissance (fig. $3 \mathrm{~A}$, $3 \mathrm{~B})$.

Les tableaux $4 \mathrm{~A}$ et $4 \mathrm{~B}$ exposent les équations de régression correspondantes, toutes significatives au seuil de I p. I 00 sauf 1'azote du stroma (significatif à 5 p. I Oo).

I,es analyses chimiques ne révélant pas de différences significatives dans le contenu en lipides intra-cellulaires, des muscles d'animaux jeunes et âgés (HELANDER, I959) il ne nous a pas semblé nécessaire d'exprimer les divers résultats en fonction de la matière sèche dégraissée.

La teneur en azote total est comprise entre 30 et $37 \mathrm{mg}$ par $\mathrm{g}$ de tissu frais dans les deux sexes. L'augmentation de cette teneur pour un même gain de poids vif, est la même chez les mâles et les femelles jusqu'à un poids vif de I ooo g, il y a ensuite un enrichissement plus marqué chez les mâles (fig. 2 A et $2 \mathrm{~B}$ ).

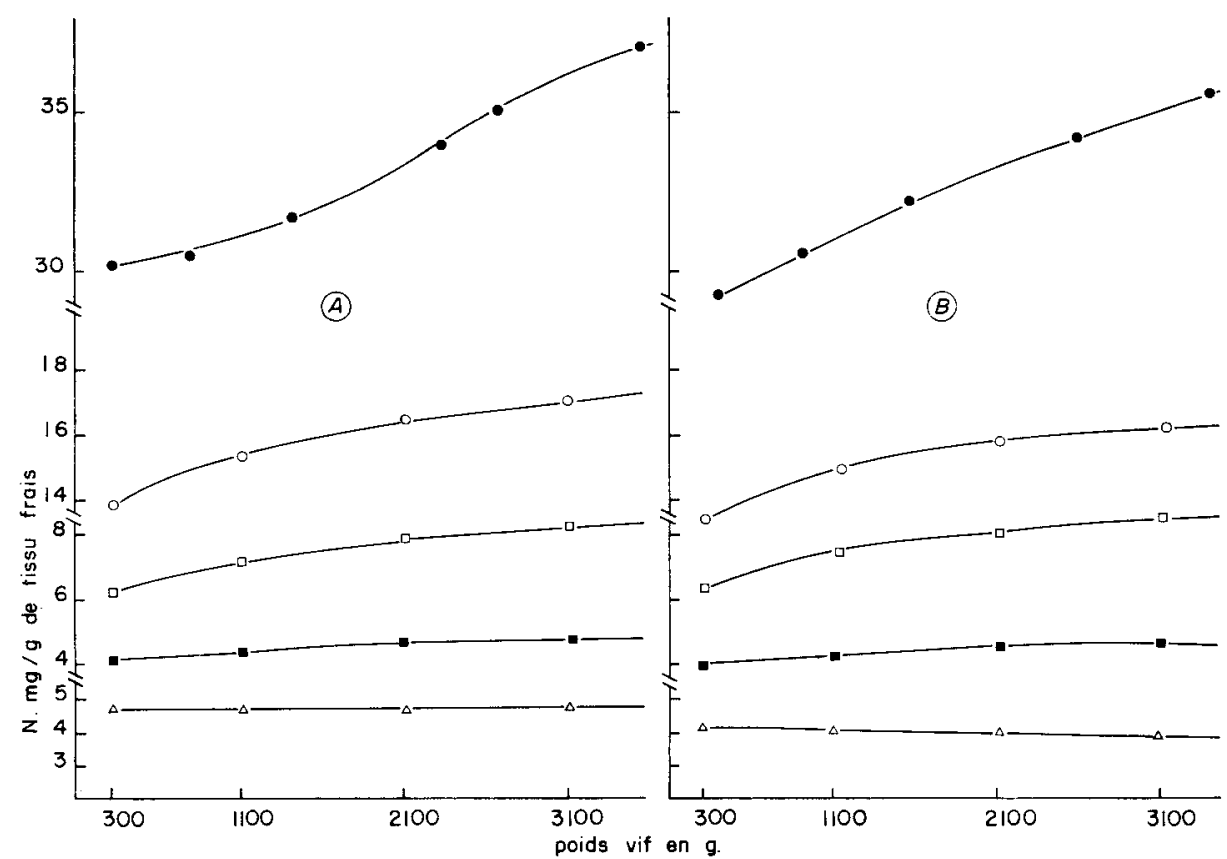

FIG. I A. - Evolution de la teneur en azote du psoas chez les lapins mâles au cours de la croissance

FIG. I B. -Évolution de la teneur en azote du psoas chez les lapins femelles au cours de la croissance

- Azote total

- Azote myofibrillaire

D Azote sarcoplasmique

- Azote non protéique

$\triangle$ Azote stroma 


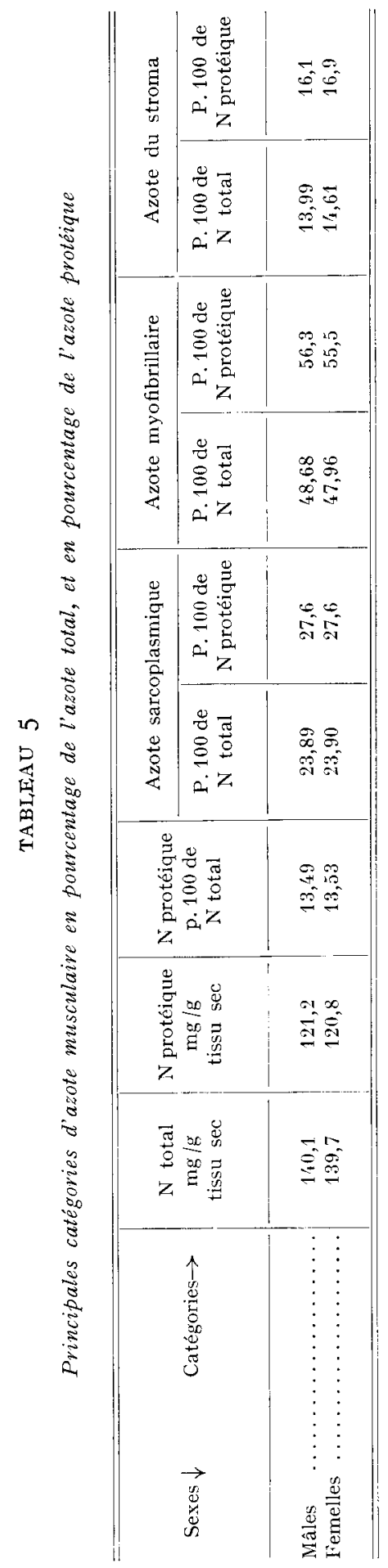


La teneur en azote myofibrillaire qui varie de $\mathrm{I}_{3}$ à $\mathrm{I} 8 \mathrm{mg}$ par $\mathrm{g}$ de tissu frais, est plus élevée chez les mâles ; cependant la variation relative au poids vif est strictement identique dans les deux sexes.

A l'opposé, la concentration en azote sarcoplasmique qui varie de 7 à Io $\mathrm{mg}$ par $\mathrm{g}$ de tissu frais est plus élevée chez les femelles. Au cours de la croissance, la variation relative de cette teneur reste importante chez les mâles alors qu'elle s'annule chez les femelles à partir d'un poids vif de 2 Ioo g.

La concentration en azote non protéique est constante dans les deux sexes (fig. $2 \mathrm{~A}, 2 \mathrm{~B}$ ) avec toutefois une variation relative plus marquée pour les femelles.

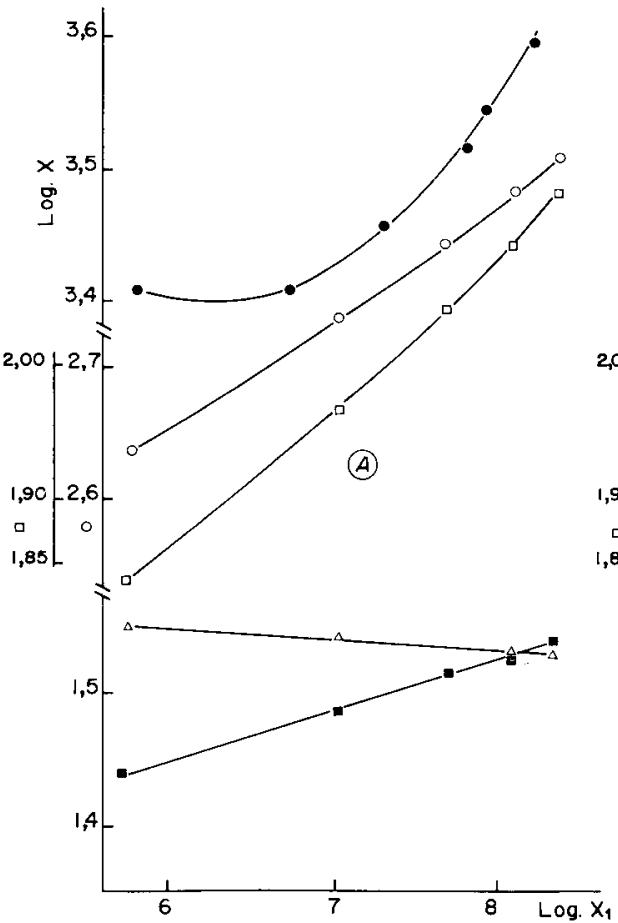

FIG. 2 A. - Évolution relative de la teneur en azote du psoas chez les lapins mâles au cours de la croissance

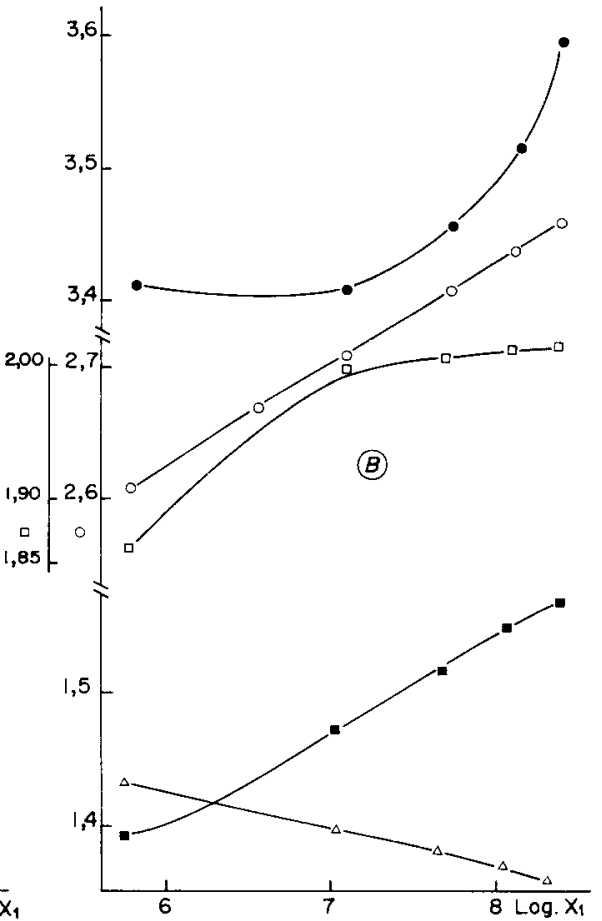

FIG, 2 B. - Évolution relative de la teneur en azote du psoas chez les lapins femelles au cours de la croissance
- Azote total
- Azote myofibrillaire
$\square$ Azote sarcoplasmique
- Azote du stroma
$\triangle$ Azote non protéique

On constate que les courbes représentatives de la concentration en matière sèche et de la concentration en azote total ont la même allure, ainsi que l'ont déjà constaté Durand et Fauconneau (I963) chez le Porc pour les concentrations en protéines et en matière sèche dégraissée.

Si l'on suit l'évolution, en fonction du poids vif, des concentrations (et de leurs variations) des différentes formes azotées ramenées au gramme de tissu sec, on obtient des droites sensiblement parallèles à l'axe des abscisses, excepté pour l'azote du stroma dont la pente est légèrement négative. 
En pourcentage de l'azote total et de l'azote protéique total, on obtient les résultats moyens présentés au tableau 5 .

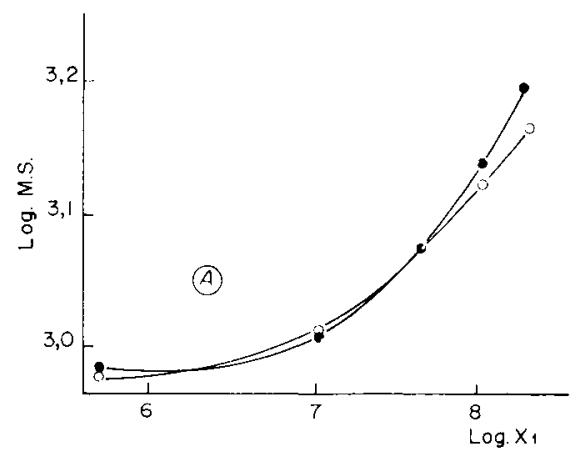

FIG. 3 A. - Evolution relative de la teneur en matière sèche du psoas chez les lapins au cours de la croissance

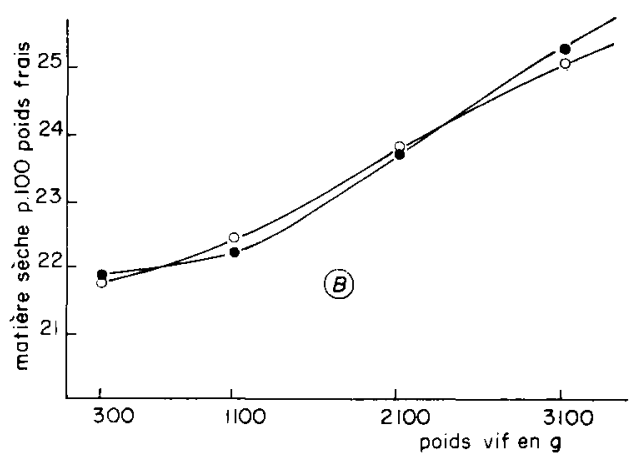

lir. 3 B. - Évolution de la teneur en matière sèche du psoas chez les lapins au cours de la croissance

femelles $o$

mâles

\section{III. - DISCUSSION}

Avec une teneur en matière sèche variant de $2 \mathrm{I}$ à $26 \mathrm{p}$. Ioo du poids frais et une teneur en azote total allant de 29 à $36 \mathrm{mg}$ par $\mathrm{g}$ de poids frais, nos résultats sont comparables à ceux obtenus en utilisant la même technique (HEIANDER, I957, I96I). De la diminution de la teneur en eau observée ici au cours de la croissance découlent les augmentations observées de la teneur en azote total et en azote des diverses catégories de protéines. Cela rejoint les observations de YANNET et DARROW (I937) chez le Chat, qui ont montré que le muscle croît surtout par expansion de la phase intracellulaire, avec diminution relative de la taille des espaces interstitiels.

Mais, d'après nos résultats ramenés au gramme de matière sèche, nous pouvons préciser qu'à partir de 3 semaines la teneur relative en azote des diverses fractions du muscle est déjà fixée chez le Lapin dans des conditions de vie normale. Ces résultats confirment ceux d'Ivanov (I959) qui ne trouve de différences dans l'évolution des quantités relatives d'azote sarcoplasmique et myofibrillaire qu'avant cet âge. Chez le Poussin, Mizuno et al. (I966) observent des changements importants dans la composition de l'azote total entre le $\mathrm{I} 7^{\mathrm{e}}$ et le $24^{\mathrm{e}}$ jour après le début de l'incubation, le pourcentage d'azote myofibrillaire augmentant et celui de l'azote sarcoplasmique diminuant. La composition biochimique du muscle semble varier de façon importante au moment où il devient fonctionnel.

Plusieurs conclusions se dégagent de ces observations :

Il est impératif d'exprimer les conśtituants biochimiques d'un tissu de façon à éliminer cette variable qu'est la teneur en eau. Pour le muscle, on peut exprimer les données en pourcentages de l'azote total. Cela permet de comparer de façon plus précise les résultats rapportés dans la bibliographie et qui ne tiennent souvent compte ni de la teneur en eau du tissu, ni de l'âge des animaux. 
Nous voyons que nos résultats ( 48 p. roo d'azote myofibrillaire) sont voisins de ceux obtenus par Hanson et HuxLEY (I957) également pour le psoas du Lapin (5I p. Ioo d'azote myofibrillaire) à l'aide d'une autre technique mais sont plus éloignés de ceux de Helander (I958) sur les muscles de la cuisse de cet animal (57 p. Ioo d'azote myofibrillaire). Par contre, nous obtenons davantage d'azote protéique sarcoplasmique et d'azote du stroma. Ces différences sont sans doute en rapport avec l'activité fonctionnelle, les muscles de la cuisse étant appelés à se contracter plus souvent et de façon plus énergique.

Nous avons mis en évidence une différence de composition faible mais significative entre les grands psoas des lapins mâles et femelles; en particulier, il y a chez les mâles davantage de protéines contractiles et moins de protéines sarcoplasmiques. A notre connaissance une différence de composition biochimique due au sexe n'avait pas encore été rapportée pour un muscle de mammifère.

Il est par ailleurs intéressant de constater que les changements d'évolution des caractères biochimiques du muscle se situent dans la zone où CANTIER et al. (I969) ont observé les changements d'allométrie des principaux tissus et organes du Lapin, ce qui rejoint les idées de NEEDHAM (I934) sur l'hétérogonie biochimique et les propositions de LAMBERT et TEISSIER (I927) sur la similarité biologique. Le " processus essentiel de la croissance se déroule selon un plan défini, reconnaissable dans la constitution de tel ou tel organisme dans un stade donné de son ontogénie ".

Enfin, il nous semble que la variable " poids vif " ne constitue pas la référence la plus satisfaisante dans les études de croissance différentielle. Il faudra donc chercher un autre critère, peut-être plus synthétique, à l'aide de techniques plus élaborées ; d'où la nécessité de connaître exactement les évolutions différentiellei des divers composants de l'organisme et de déterminer leur importance respective au cours du développement.

$$
\text { Rę̧u pour publication en férrier } 1970 .
$$

\section{REMERCIEMENTS}

Les calculs ont été effectués sur l'IBM I I 30 de la Station de Biométrie de C. N. R. F. ; nous remercions MM. Tomassone et Millier pour les conseils et l'aide qu'ils ont bien voulu nous apporter.

\section{SUMMARY}

BIOCHEMICAL HETEROGONY OF NITROGENOUS COMPONENTS

OF THE PSOAS MAJOR MUSCLE DURING POST-NATAL GROWTH OF RABBITS

Composition of the psoas major was studied in male and female rabbits of ages ranging from 3 to 26 weeks. The study concerned the nitrogen contents of the following fractions : myofibrils. sarcoplasm, stroma and non-protein nitrogen. These were estimated by the technique of HELANDER. Dry matter content of the muscle was estimated also.

The development of the relative changes in concentration of each nitrogen fraction was studied with a logarithmic polynomial model.

The dry matter content increased during life, from about 2 I.5 to about 26.0 p. Ioo. 
The relative contents of nitrogen seem to remain unaltered after the age of 3 weeks in the rabbit (table 5).

There is a small but significant difference in nitrogen content of the psoas major between males and females; males had more myofibrillar nitrogen and less sarcoplasm nitrogen.

\section{RÉFÉRENCES BIBLIOGRAPHIQUES}

Bendall J. R., I966. in : BRISKey E. J. The physiology and biochemistry of muscle as a food. 7-I6, Univ. Wisconsin Press Londres.

Brody S., r945. Bioenergetics and growth. Reinhold, New York, 1023 p.

Cantier J., Vezinhet A., Rouvier R., Dauzier L., ig69. Allométrie de croissance chez le Lapin (Oryctolagus cuniculus). Ann. Biol. anim. Biochim. Biophys., 9, 5-39.

CREPAX P., 1952. Étude électrophorétique d'extraits de muscles doués de différentes propriétés morphologiques et fonctionnelles. Biochim. Biophys. Acta, 9, 385-398.

Dagnelie P., I964. Sur la détermination du nombre de répétitions en vue de l'estimation d'une moyenne Biometr. Praxim., 5, I 7 7-I35.

Dickerson J. W. T., rg6o. The effect of growth on the composition of avian muscle. Biochim. J., 75 33-37.

Dickerson J. W. T., Widdowson E. M., 1960. Chemical changes in muscle during development. Biochim. J., 74, 247-257.

Durand G., Fauconneau G., Penot E., I967. Évolution de la teneur en acides nucléiques et en protéines du muscle chez le Porc au cours de la croissance postnatale. C. R. Acad. Sci. Paris, 264, I 640-I 643 .

Gordon E. E., Kowalski K., Fritts M., ig66. Muscle proteins and DNA in rat quadriceps during growth. Am.J.Physiol, 210, I033-1040.

Gunther B., Guerra F., I955. Biological similarities. Acta Physiol. latinoam., 5, I69-I86.

Gunther B., Guerra F., I957. Biological similarities. Acta Physiol. latinoam, 7, 95.

HANSON J., HUXLEY H. F., r957. Quantitatives studies od the structure of cross striated myofibrils. II. Investigations by biochemical techniques. Biochim. Biophys. Acta, 23, 250-26o.

Helander E., r957. On quantitative muscle protein determination. Acta Physiol. scand., Suppl. 141, 9-95.

Helander E., I958. Adaptative muscular "allomorphism ". Nature, 182, I035-1036.

Helander E,, 1959. Fat content skeletal muscular tissue. Acta Morphol. Res, scand., 2, $230-254$.

Helander E., r96r. Exercise and muscle protein composition. Biochem. J., 78, 478-482.

Herrman H., Nicholas J. S., I948. Quantitative changes in muscle protein fractions during rat development. J. exp. Zool., 107, 165.

Ivanov I. I., 1967. Organic substances of the striated muscle. Symp. Biol. Mung., 8, 89-124.

Lambert R., Teissier G., I927. Théorie de la similitude biologique. Ann. Physiol. Physicochim. biol., 3, 2 I2-246.

LAWRIE R. A., I96I. Systematic analytical differences between psoas major and longissimus dorsi muscles of cattle. Br. J. Nutr., 15, 453-456.

Millier C., 1968. Analyse de variance à un facteur contrôlé. Station de Biométrie C. N. R. F., Programme FORTRAN 68 033. Nancy.

Mizuno T., Hikami Y., Sumita K., $x_{966}$. The accumulation of nucleic acid and protein in the skeletal muscle of chicks during embryonic and post embryonic development. Jap. J. vet. Sci., 37, $47 \mathrm{I}-477$.

Moss F. P., Leibholz J., Simmonds R. A., ig68. The growth and composition of skeletal muscle in the chicken. III. The variation with age of certain protein nitrogen fractions. Poult. Sci., 4\%, 475-479.

Moss F. P., Srmmonds R. A., McNARY H. W., I964. The growth and composition of skeletal muscle in the chicken. Poult. Sci., 43, ro86-ıogo.

Needham J., I933. Chemical heterogony and the ground-plan of animal growth. Biol. Rev!, 9, 79-109,

Prud'hon M., I967. L'appétit du Lapin alimenté à sec. Bull. tech. Inform. Ingrs. Servs. agric., 219. 383-398.

Ricard F.-H., Rouvier R., i965. Étude des mesures de conformation du Poulet. I. Analyse statitique préliminaire concernant le poids et 13 mensurations corporelles du Poulet vivant. Ann. Zootech., 14, $\mathrm{I} 9 \mathrm{I}-2 \mathrm{I} 2$.

Scharpf L. G., MARION W. W., 1964. Extraction of fibrillar and sarcoplasmic proteins of turkey muscle. J. Food. Sci., 29, 303-306.

StahL W. R., I962. Similarity and dimensional methods in Biology. Science, 137, 205-212.

Tomassone R., 1967. Regression progressive multiple. Station de Biométrie C. N. R. F., Programme FORTRAN 67009. Nancy.

YANNET H., DARRow D. C., 1937. The effect of growth on the distribution of water and electrolytes in brain, liver and muscle. J. biol. Chem., 123, 295-305. 